\section{A Novel FIB Method for Preparing Three Dimensional TEM Specimens}

\author{
Nathan Wang \\ Cypress Semiconductor Corp., San Jose, CA \\ nxw@cypress.com
}

\section{Introduction}

Presently, there is an increasing demand for multi-view TEM observation of semiconductor device failure locations where the feature size of interest is smaller than the specimen thickness, and where the thin section has other features that may have high $\mathrm{Z}$ materials that obscure the site of interest in the TEM [1]. Therefore, different viewing angles are necessary - such as a cross-section view of a defect-containing planar thin specimen. A major obstacle for the preparation of three dimensional specimens of this nature is specimen preparation. Great care must be taken when preparing a thin specimen from an already thin existing TEM specimen. In this article, three-dimensional TEM specimen preparation refers to the creation of a single specimen that contains adjacent, multiple views, at different orientations with respect to each other, e.g., planar and cross section images.

This technique was originally developed for cases where cross sectional viewing was desired, but a precise target location was unavailable; so a plan view TEM specimen provided the precise location for a subsequently prepared cross-section specimen [2]. The method is extended in this article to the creation of a second cross section, perpendicular to a first cross section: a cross section of a cross section.

\section{Main Issues}

There are two key steps in 3-D specimen preparation: 1.) creating a thin film at the target location that is electron transparent,

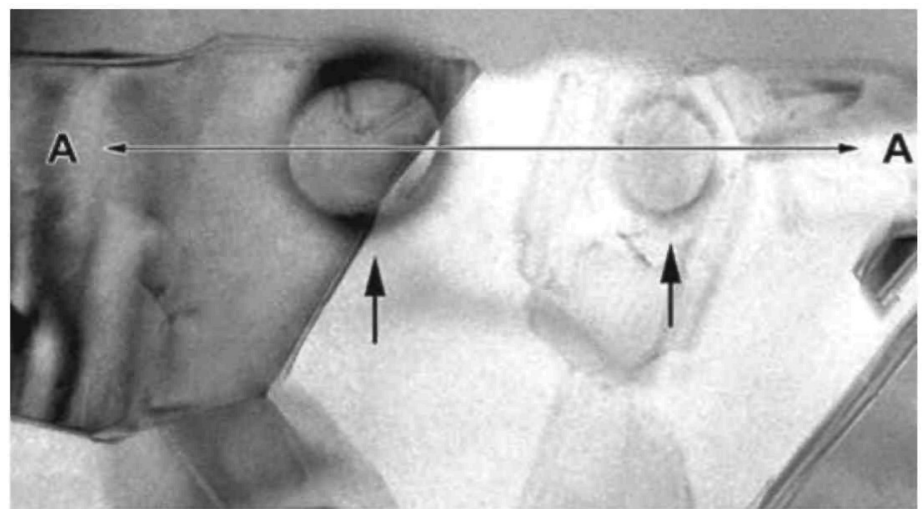

Plane-view TEM image

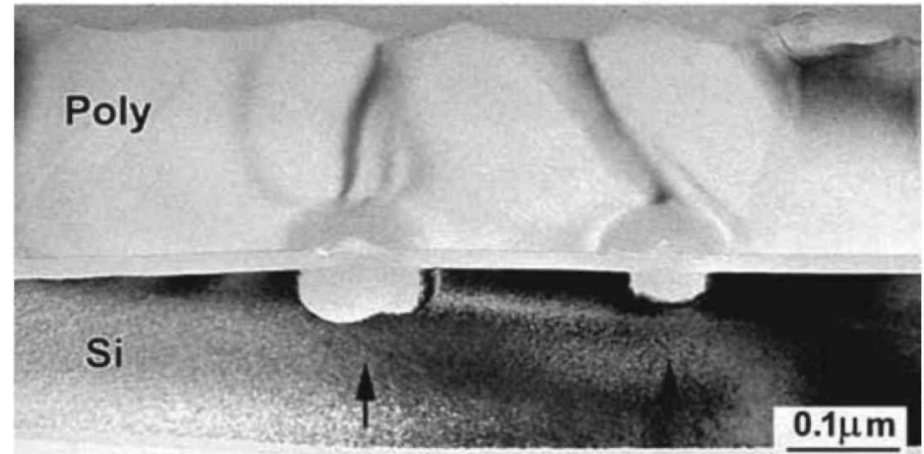

Cross-sectional TEM image at location A-A

Figure 1:3D TEM image that was done in 1999: It revealed gate oxide pinholes.

and then 2.) tilting a portion of the specimen 90 degrees relative to the original specimen orientation so that the thin target area will be facing the electron beam in the TEM in a direction 90 degrees removed from the first viewing direction.

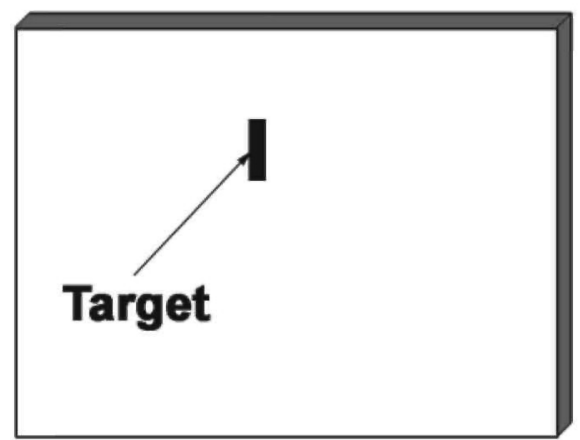

Fig. 2A) Mark and deposit a protective layer at the target

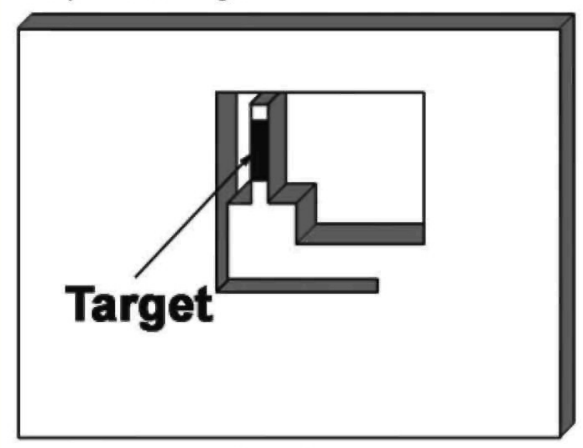

Fig. 2D) Make a fine cut to prepare a thin membrane at the target

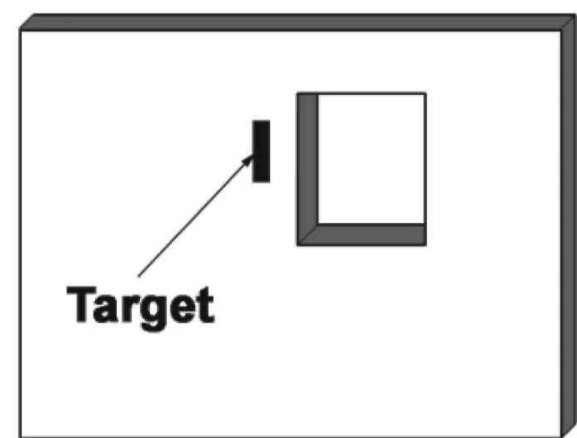

Fig. 2B) Create a window on one side of the target

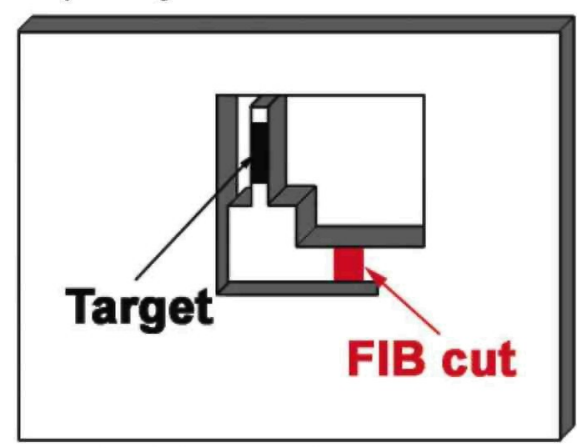

Fig. 2E) FIB erode the connection area to bend the small piece containing the target.

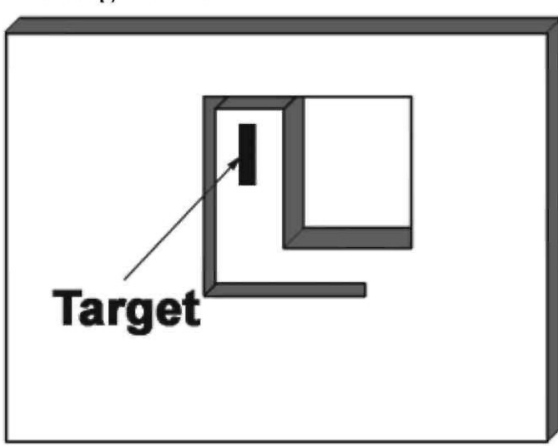

Fig. 2C) Isolate the target by cutting trenches

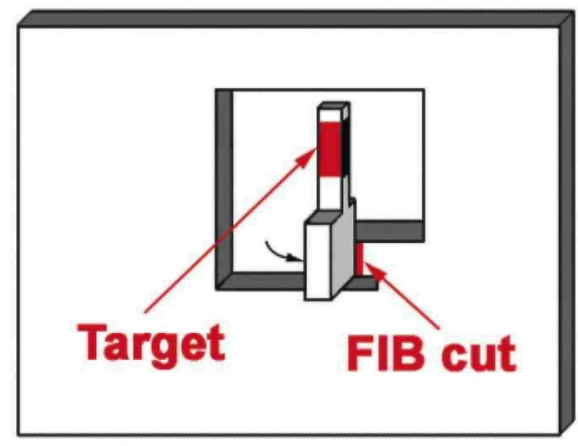

Fig. 2F) Stop cutting when the little piece is bent to about 90 degrees

Figure 2: Diagrams illustrate the specimen preparation procedure. The gray tint is meant to depict the walls of the FIB cuts perpendicular to the surface. The window in figure $2 B$ is a perpendicular opening from top to bottom of the thin substrate. 


\section{It's the same with}

\section{microanalysis...}

\section{you want your \\ supplier to always \\ be there for you.}

At EDAX, we take enormous pride in providing today's best microcharacterization technologies. But this is not the end. In fact, installing your system is just the beginning of your relationship with EDAX.

We are equally proud of our unparalleled global support for all our customers. EDAX provides frequent, in-depth training classes, skilled service engineers on call to assist you and scientists to advise you in your specific applications.

Next time you are in the market for X-ray microanalysis, EBSD or micro-XRF systems, come to EDAX. We will not let go of you after the sale is made.

Discover what good service and support are all about at: www.edax.com/thinkEDAX 

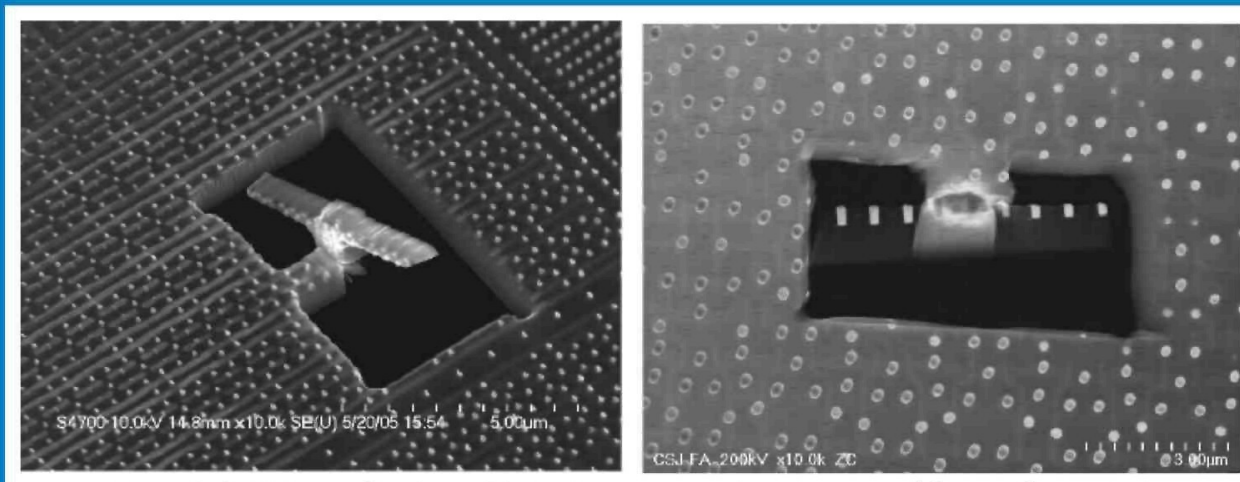

Figure 3: SEM image showing a 3D specimen cross section view created from a planar specimen that is leaning to one side (left), Specimen tilting with a TEM holder can overcome this problem. TE mode dark field image is shown on right.

The first successful 3-D specimen was made in 1999

at Precision TEM (figure 1.). A mechanical polishing technique was utilized for both steps in the preparation process with the result that the resulting two views revealed gate oxide pinholes. Since then, researchers have developed other techniques [2] [3] [4] [5] [6] [7] that either eliminate or minimize skilled mechanical polishing operations for the second (tilting) step. These include the Omniprobe method [2] [4], the lift-out method [5], the vertical grid transferring method [6], and the saw method [7]. All of these methods take advantage of a FIB tool's precise cutting capability to create a thin membrane at the target area. One critical distinction between the referenced methods and the method presented in this article is that, in the referenced methods, specimen tilting to view planar specimens in cross section is accomplished via the transfer of the specimen to a second grid for the specimen preparation second (tilting) step. The transferring process can be complicated and result in a low yield of 3D specimens.

Directly tilting a portion of the specimen on the original grid through 90 degrees will simplify greatly the preparation process and increase the yield of 3-D specimens due to the fact that it avoids the specimen transferring step. This can be done by using a FIB cut to bend a portion of the specimen silicon through 90 degrees on the original grid. Both steps: thin membrane creation at a target and local specimen bending are done sequentially in the same FIB chamber and on the same grid.

\section{Tilting Process Steps}

It is well known that silicon is a brittle material that breaks when it is bent. However, this is true only for a "thick" silicon specimen. Many FIB users have experienced the phenomenon that a small piece of silicon may bend toward the beam during FIB thinning and especially when the specimen is being cut free of its substrate. This fact illustrates the fact that silicon is very flexible when thin. The force that causes the bending up towards the beam is believed to be electrostatic in nature. We can take advantage of this phenomenon to controllably bend a small piece of thin specimen at the target site in an original specimen by FIB cutting it so that FIB-induced specimen tilting can be realized. The following is the detailed description of the procedure.

First, a FIB is used to mark the target location and deposit a protective layer on top of the target if necessary (figure 2A). Then, on one side of the target, create a rectangular window. This window will be the viewing area for the final specimen after the target tilting operation (figure $2 \mathrm{~B}$ ). Cut trenches around the target to isolate the target location from the bulk of the thin specimen substrate.

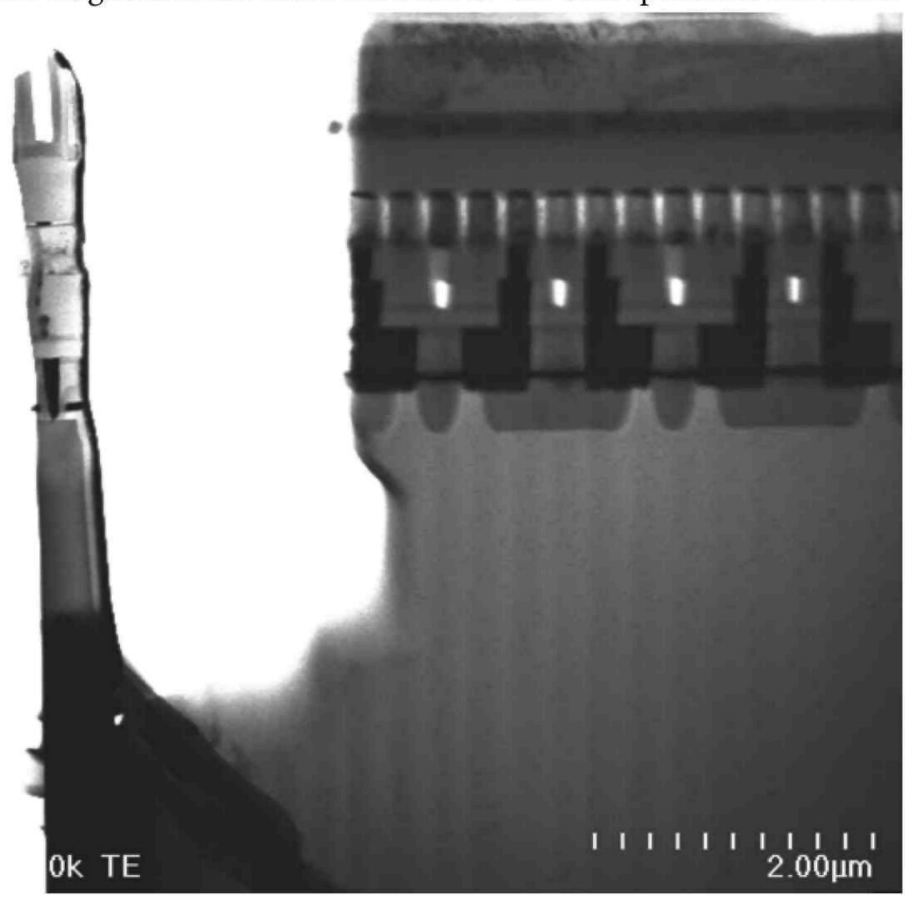

Figure 4: A low magnification TE mode image shows the tilted specimen (on left) and the untilted part (on right). Both parts are nearly perpendicular to each other.

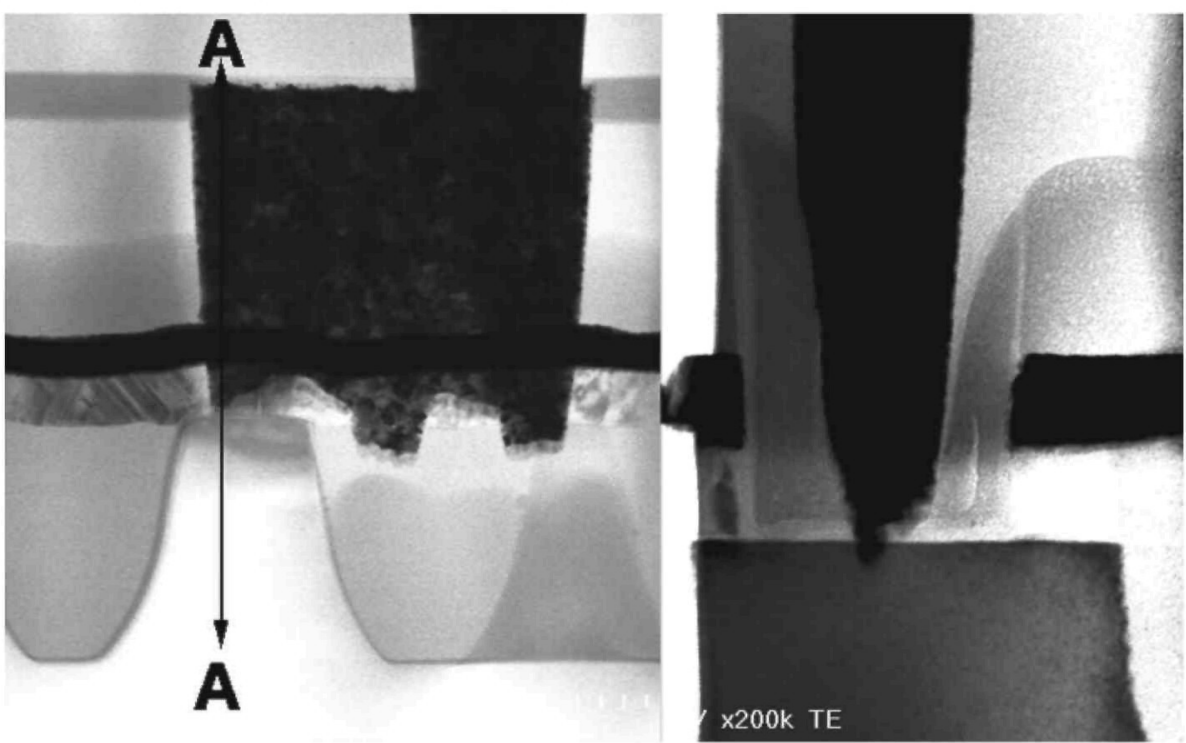

Figure 5: A high magnification image of the figure 4 specimen: the left view shows $x$-direction cut and the right one shows the $3 D$ image at location $A-A$. 
One end is left uncut to maintain a connection between the small, target-containing, piece and the thin specimen substrate (figure 2C). A fine cut is then made to form a thin electron-transparent membrane at the target area for subsequent TEM viewing (figure 2D). The next cut is at the connection area between the small piece and the main part (figure 2E). This cut is the most important step because it determines the bending angle of the small piece of silicon relative to the main part of the thin specimen substrate.

Before this final cutting operation, the substrate silicon lattice keeps the small target piece attached and co-planar with respect to the main part of the specimen substrate. During this final cut, the silicon lattice between the two parts is eroded to the point that the target-containing piece begins to bend up toward the beam. The specimen movement can be seen and monitored in the previously FIB-created window. Since the amount of erosion of the silicon lattice via FIB cutting, controls the bending angle of the targetcontaining piece, repeated cutting operations may be necessary to achieve the desired angle, which is about 90 degrees with respect to the original thin substrate. In practice, a perfect 90 degree tilt is hard to achieve and a little leaning toward the side may also occur. One example of a leaning specimen is shown in Figure 3. However, a deviation off 90 degrees and a small amount of perpendicular leaning is of no consequence and can be easily adjusted away via the TEM's goniometer stage during analysis. A FIB deposit in the bending hinge area can be performed to lock the final tilt position. The final specimen shape is shown in figure $2 \mathrm{~F}$. In this way the target area is successfully tilted and a 3-D TEM specimen is formed.

\section{Results}

The method is very simple. The specimen preparation procedure, post initial view specimen creation, including thin membrane creation and specimen tilting, is accomplished and monitored in the FIB, and a near $100 \%$ success rate is easily achievable. The whole process can be done within 15-30 minutes.

An example is shown in figures 4 and 5 that reveal contact blocking failure. Figure 4 is a low magnification STEM image that shows the structures in 3-D mode. In this case we show a cross section created from a cross section. The tilted, target-area, cross section is on the left and the untilted, cross section substrate is on the right. Figure 5 is a $3-\mathrm{D}$ image comparison: The left image is

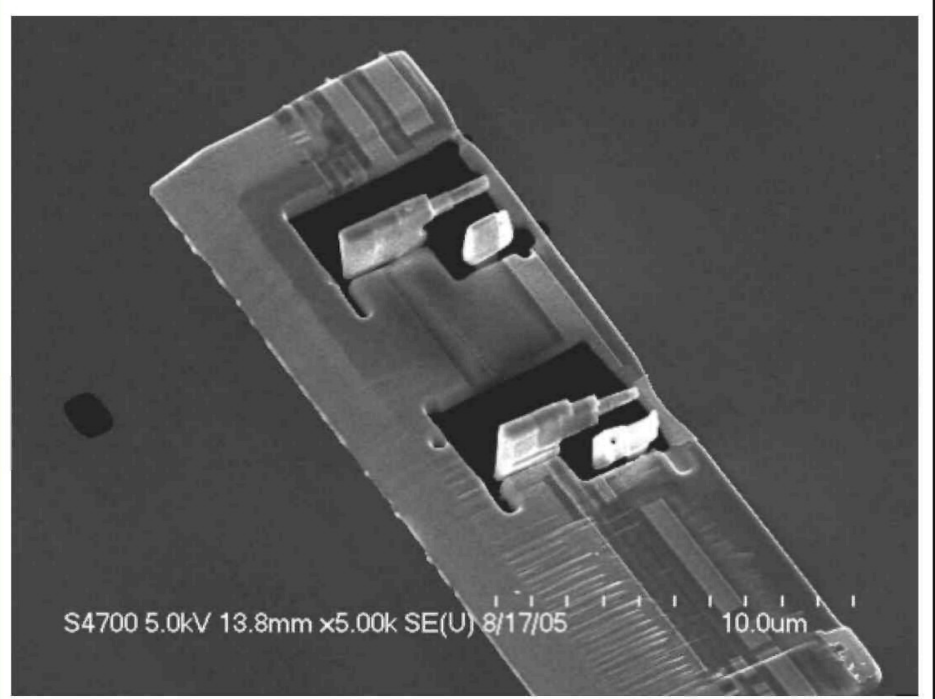

Figure 6: Two 3-D specimen regions cut into a FIB Lift-Out specimen resting on a carbon film on a TEM grid. from the original untilted cross section and reveals partial contact blocking. The right image is from the cross section tilted 90 degrees with respect to the left figure and reveals the failing root cause of poor spacer nitride.

The first, initial thinning preparation step, can be accomplished by a variety of methods. Initial thinning for the figure 3 example was performed by tripod polishing, which will result in a very large area for step 2 processing. The initial preparation of the specimen in figures 4 and 5 was via an ex-situ FIB "Lift-Out" specimen preparation protocol, with the section FIB-tacked to a tripod polished specimen. The two 3-D specimens in figure 6 were cut into a single in-situ FIB "Lift-Out" specimen on a carbon film coated TEM grid.

\section{References}

[1] K. Mcllwrath and N. Wang, ISTFA 2004, (2004), 320

[2] N. Wang and Sabbas Daniel, Microelectronic Failure Analysis Desk Reference 2002 suppl., ASM international, Materials Park, OH, (2002), pp.21-28

[3] C.K. Liu et al, ISTFA 2002, (2002), 313

[4] T. Yaguchi, et al., Microscopy Today, 12 (6), (2004), 26

[5] J. C. Lee, et al., Microelectronics Reliability 41, (2001), 1551

[6] N. Wang, et al., Microsc. Microanal. 11(Suppl 2), (2005), 2100.

[7] R. B. Irwin, et al., Microscopy Today, 13 (1), (2005), 26

\section{NORTHWESTERN UNIVERSITY RESEARCH ASSOCIATE - NANOTECHNOLOGY}

Established in 1851, Northwestern University has an excellent reputation as a leader in higher education, offering world class academic, research and professional opportunities. Northwestern University Atomic and Nanoscale Characterization Experimental (NUANCE) Center seeks a seasoned and high technology-oriented Research Associate to oversee a surface science facility. This facility is part of a larger center (www.nuance.northwestern.edu) that houses four high-level materials characterization facilities and serves over 500 users.

Responsibilities Include:

- Trains users, graduate and undergraduate students and some external business customers, on the instruments and develops their skills

- Provides technical support and collaborative assistance to users on their projects

- Maintains facility and instruments

- Collaborates with faculty on research and publication of results

- Conducts independent research

- Manages a budget of $\$ 150,000$

Minimum Qualifications Include:

- Ph.D. in Physical Sciences or Engineering

- One year of laboratory experience

Solid knowledge of and hands-on experience in comprehensive characterization with surface science techniques such as XPS, SIMS, FTIR and SPM

- Strong written and verbal communication skills

Preferred Qualfications:

- 3-5 years of relevant laboratory experience

- Experience with instructional design and delivery of technical material to students

- Documented research and scientific publications

Candidates are requested to send resume, a publication list and three reference letters to: Email: r-mccullough@northwestern.edu Northwestern University is an Equal Opportunity, Affirmative Action Employer. Members of historically underrepresented groups are strongly encouraged to apply. 